\title{
MRI Findings Suggestive of Herpes Simplex Encephalitis in Patients with Anti-NMDA Receptor Encephalitis
}

W e read with great interest the recent article by Zhang et $\mathrm{al}^{1}$ regarding brain MR imaging characteristics of 53 patients with anti- $N$-methyl-D-aspartate (NMDA) receptor encephalitis. In their important analysis, the authors describe 4 distinct imaging patterns: normal MR imaging findings (type 1, 53\% of patients), isolated hippocampal involvement (type 2, 13\%), other brain lesions without hippocampal involvement (type 3, 13\%), and other brain lesions with hippocampal involvement (type 4 , $21 \%)$.

The authors provide representative images of these 4 imaging types. The image example for type 4 (lesions in both the hippocampus and other brain areas, Fig $1 D 1$ and D2) shows an asymmetric T2/FLAIR hyperintense presentation of both medial temporal lobes and large parts of the left lateral temporal lobe as well as bilateral frontal, insular, and cingulate involvement. In our experience, this MRI pattern is very atpyical for anti-NMDA receptor encephalitis, for which we have observed much more circumscribed hippocampal T2/FLAIR hyperintense signal alterations, and only in a few patients, ${ }^{2,3}$ but would be compatible with herpes simplex encephalitis (HSE) imaging findings.

Recently, it has been shown that up to $20 \%$ of patients with HSE develop postviral anti-NMDA receptor encephalitis. ${ }^{4}$ These patients have HSE followed by autoimmune encephalitis with NMDA receptor autoantibodies weeks to months after initial recovery. In light of these findings and the imaging pattern suggestive of HSE in the article by Zhang et al, ${ }^{1}$ we would like to suggest that the presented patient did not have isolated anti-NMDA receptor encephalitis but rather HSE followed by anti-NMDA receptor encephalitis. In our opinion, it would be most important to discriminate these 2 patient groups, and it would therefore be very interesting to know how many patients with type 4 have an imaging pattern that would be compatible with HSE MR imaging findings. We would like to encourage the authors to present MR imaging examples of all group 4 patients in an article supplement. This would be of great interest to the readers and of immense help in clinical practice.

http://dx.doi.org/10.3174/ajnr.A5788
If some patients with type 4 presented by Zhang et $\mathrm{al}^{1}$ indeed have post-HSE anti-NMDA receptor encephalitis, it would also affect the interpretation of the correlation analysis, in which a worse outcome was associated with hippocampal lesions. While most patients with anti-NMDA receptor encephalitis recover quite well and frequently do not show any specific MR imaging changes, most patients with HSE have persistent cognitive impairment associated with hippocampal/medial temporal lobe damage and corresponding MR imaging findings. Hence, including patients with post-HSE anti-NMDA receptor encephalitis in this correlation analysis could bias the analysis toward an association of hippocampal involvement with poor outcome. It would therefore also be very interesting to reconsider this correlation analysis.

Disclosures: Carsten Finke-UNRELATED: Grants/Grants Pending: Deutsche Forschungsgemeinschaft, German Ministry for Education and Research. * *Money paid to the institution.

\section{REFERENCES}

1. Zhang T, Duan Y, Ye J, et al. Brain MRI characteristics of patients with anti-N-methyl-D-aspartate receptor encephalitis and their associations with 2-year clinical outcome. AJNR Am J Neuroradiol 2018;39:824-29

2. Heine $J$, Prüss $H$, Bartsch $T$, et al. Imaging of autoimmune encephalitis: relevance for clinical practice and hippocampal function. Neuroscience 2015;309:68-83 CrossRef Medline

3. Bacchi S, Franke K, Wewegama D, et al. Magnetic resonance imaging and positron emission tomography in anti-NMDA receptor encephalitis: a systematic review. J Clin Neurosci 2018;52:54-59 CrossRef Medline

4. Prüss H. Postviral autoimmune encephalitis: manifestations in children and adults. Curr Opin Neurol 2017;30:327-33 CrossRef Medline

(D) M. Scheel

NeuroCure Cluster of Excellence and NeuroCure Clinical Research Center Department of Neuroradiology Charité-Universitätsmedizin Berlin Berlin, Germany (D) C. Finke

Department of Neurology Charité-Universitätsmedizin Berlin Berlin, Germany Berlin School of Mind and Brain, Humboldt-Universität zu Berlin Berlin, Germany 